\title{
A Comparative Study of Parallel Programming Frameworks for Distributed GPU Applications
}

\author{
Ruidong $\mathrm{Gu}^{1}$, Michela Becchi ${ }^{1}$ \\ Department of Electrical and Computer Engineering \\ ${ }^{1}$ North Carolina State University \\ \{rgu, mbecchi\}@ncsu.edu
}

\begin{abstract}
Parallel programming frameworks such as MPI, OpenSHMEM, Charm++ and Legion have been widely used in many scientific domains (from bioinformatics, to computational physics, chemistry, among others) to implement distributed applications. While they have the same purpose, these frameworks differ in terms of programmability, performance, and scalability under different applications and cluster types. Hence, it is important for programmers to select the programming framework that is best suited to the characteristics of their application types (i.e. its computation and communication patterns) and the hardware setup of the target high-performance computing cluster.
\end{abstract}

In this work, we consider several popular parallel programming frameworks for distributed applications. We first analyze their memory model, execution model, synchronization model and GPU support. We then compare their programmability, performance, scalability, and load-balancing capability on homogeneous computing cluster equipped with GPUs.

\section{KEYWORDS}

Parallel computing, Distributed applications, Homogeneous cluster.

ACM Reference format:

Ruidong Gu, Michela Becchi. 2019. A Comparative Study of Parallel Programming Frameworks for Distributed GPU Applications. In Proceedings of ACM Computing Frontiers conference, Alghero, ITALY, April 30-May 2, 2019 (COMPUTING FRONTIERS'19). ACM, New York, NY, USA, 6 pages. https://doi.org/10.1145/3310273.3323071

\section{Introduction}

Parallel programming frameworks such as MPI [1], Partitioned Global Address Space (PGAS) languages (e.g., OpenSHMEM [2], UPC [3], Chapel [4], and X10 [5]), and Cham ++ [6] have been widely used in many scientific domains (from bioinformatics, to computational physics, chemistry, among others) to implement distributed applications. With the widespread adoption of GPUs in high performance supercomputers (e.g., Piz Daint, Tsubame 3.0, Titan, Tianhe-2A), GPU integration has also become an important feature of programming frameworks for distributed systems. More recently,

Permission to make digital or hard copies of all or part of this work for personal or classroom use is granted without fee provided that copies are not made or distributed for profit or commercial advantage and that copies bear this notice and the full citation on the first page. Copyrights for components of this work owned by others than ACM must be honored. Abstracting with credit is permitted. To copy otherwise, or republish, to post on servers or to redistribute to lists, requires prior specific permission and/or a fee. Request permissions fromPermissions@acm.org.

CF '19, April 30-May 2, 2019, Alghero, Italy

(C) 2019 Association for Computing Machinery.

ACM ISBN 978-1-4503-6685-4/19/05...\$15.00

https://doi.org/10.1145/3310273.3323071 there have been several efforts focusing on the design and development of novel parallel programming frameworks aimed to increase programmer productivity by delegating complex data management and synchronization tasks to the runtime system. Examples include Legion [7], StarPU [8] and StarSs [9].

While they have the same purpose, these frameworks differ in terms of programmability, performance, and scalability under different applications and cluster types. Hence, it is important for programmers to select the programming framework that is best suited to the characteristics of their application types (i.e., computation and communication patterns) and the hardware setup of the target computing cluster. The main goal of this work is to provide a comparative analysis of relevant parallel computing frameworks for distributed applications and highlight their strengths and weaknesses. To this end, we select three established and one emerging library-based programming frameworks for distributed systems, namely: MPI, OpenSHMEM, Charm++ and Legion. We study four core features of these frameworks: memory model, execution model, synchronization model and GPU support. We then perform an extensive evaluation of these frameworks covering three aspects: programmability, performance and scalability on homogeneous cluster.

In this paper, we make the following contributions.

- We provide a comparative analysis of three established and one new proposed library-based parallel programming frameworks for distributed applications, namely: MPI, OpenSHMEM, Charm++ and Legion. Our analysis covers execution model, memory model, synchronization model and GPU support.

- We analyze the effect of these features on programmability and performance.

- We develop a benchmark suite that includes the implementation of applications with various computation and communication patterns (i.e., N-body simulation, dense matrix multiplication, Needleman-Wunsch sequence alignment, Himeno and a Circuit Simulator) using the considered programming frameworks. Our benchmark suite is accessible: $<$ https://github.com/raydongpub/Muti_framework_benchmark _suite.git $>$.

- We use this benchmark suite to evaluate the performance and scalability of the four programming frameworks in homogeneous hardware setting.

\section{Background}

This section provides background information on the programming frameworks considered in our analysis.

Message Passing Interface (MPI) is the most popular standard for implementing distributed applications based on the message-passing communication model. While the first version of the standard offers only two-sided communication primitives (such 
as send and receive), its second version (MPI-2) includes primitives for one-sided communication and dynamic process creation. There are different library implementations of the MPI standard. In this paper, we use OpenMPI and MPI-2.

OpenSHMEM [2] is a standardized parallel programming API based on the Partitioned Global Address Space (PGAS) model. PGAS provides a shared memory address space abstraction to applications and includes three main features: 1) a local-view programming style, 2) a global address space, and 3) one-sided communication. Along with the API specification, OpenSHMEM includes a reference implementation. The PGAS model is implemented by several programming languages, like Unified Parallel C (UPC [3]), Chapel [4], and X10 [5]. Since we focus on library implementations, we selected OpenSHMEM as a representative PGAS framework.

Charm++ [6] is an object-oriented parallel programming framework including a $\mathrm{C}++$ library API and a runtime system. Charm ++ represents tasks as objects that communicate by exchanging messages and are scheduled to execute on different processors, and it provides object migration as a load balancing mechanism [10]. The popular NAMD molecular dynamics code [11] is implemented using Charm++.

Legion [7] is a data-centric programming system developed in 2012 by researchers at Stanford University. Originated from a relatively recent research project, Legion is less established and has a more limited user base than the other frameworks considered. Nevertheless, we have selected it for our study as example of datacentric, library-based programming framework. Legion provides API primitives allowing the definition of data properties such as data structure, partitioning, access privileges and coherence, as well as abstractions to define tasks and their mapping to the underlying hardware resources. Legion's runtime system then includes mechanisms to extract parallelism from the application and orchestrate the necessary data transfers in accordance with application-specific data properties.

\section{Programmability Analysis}

In this section, we study how MPI, OpenSHMEM, Charm++ and Legion compare in terms of programmability. In particular, we analyze how the memory model, the synchronization model, the execution model and the GPU support provided by these programming frameworks affect the code complexity. In our analysis we consider eight code variants: MPI_2S and MPI_1S (basic MPI implementations using two- and one-sided communication, respectively), MPI_2S_DYN and MPI_1S_DYN (MPI code variants using dynamic process creation to provide load balancing), OSHM (OpenSHMEM), CHARM_NLB and CHARM_LB (Charm++ code variants without and with load balancing support), and LEGION.

We implemented the five applications described in Section 4.1 using these code variants. Table 1 reports the line of codes (LOC) of each implementation. As can be seen, in the case of MPI, while onesided communication allows a slight LOC reduction, the

Table 1 Lines of code

\begin{tabular}{|c|c|c|c|c|c|}
\hline & DMM & HIMENO & NBODY & NW & CCT \\
\hline MPI_2S & 280 & 671 & 626 & 399 & 611 \\
\hline MPI_1S & 278 & 662 & 623 & 395 & 596 \\
\hline MPI_DYN_2S & 465 & 724 & 712 & 573 & 1155 \\
\hline MPI_DYN_1S & 433 & 693 & 696 & 554 & 1128 \\
\hline OSHM & 276 & 831 & 618 & 357 & 544 \\
\hline CHARM_NLB & 259 & 620 & 560 & 260 & 527 \\
\hline CHARM_LB & 269 & 631 & 573 & 270 & 539 \\
\hline LEGION & 480 & 1102 & 857 & 666 & 1162 \\
\hline
\end{tabular}

communication model does not affect significantly the code length. On the other hand, adding load balancing via dynamic process creation to the MPI implementations causes the LOC to increase, in some cases significantly. As could be expected, the code length of the OpenSHMEM-based implementations is generally very similar to that of the static MPI code variant based on one-sided communication (MPI_1S). Furthermore, Charm++ leads to the most compact code, and adding load balancing has a minor effect on code length. Finally, Legion results in all cases in the least compact code.

While LOC represents an intuitive, high-level metric to compare different code variants, if taken alone it provides limited information and insight into the programmer productivity provided by different programming frameworks. To better understand and characterize the programmability of MPI, OpenSHMEM, Charm++ and Legion, we now look more closely into how the main features of the considered frameworks affect code size and complexity.

Memory and synchronization model - The memory model is the view of the memory space provided by the programming framework to applications, and it affects the way programmers perform workload partitioning and describe the communication patterns within the application. The synchronization mechanisms required to handle data and task dependences can be explicit (i.e., directly available to the programmer), or implicit (i.e., handled by the runtime system). MPI and OpenSHMEM provide explicit application-level synchronization primitives (such as barrier synchronization) and synchronization mechanisms embedded in other primitives (e.g., collective communication). As a consequence, it is the programmers' responsibility to judiciously use the available synchronization mechanisms in their application. To improve programmer productivity, Charm++ provides high-level synchronization primitives and delegates to its runtime system the task of using low-level synchronization mechanisms to satisfy the tasks and data dependences within the application.

While MPI-1 provides a disjoint memory address space, MPI2, OpenSHMEM, and Legion provide a unified memory address space, which enables direct access to data objects for all PEs no matter where the PEs and the data objects reside. Charm++ adopts a hybrid model: while PEs operate on local variables private to them, they share a global name space. For data exchanges, MPI-1 provides two-sided communication primitives (e.g., send and receive); MPI-2 and OpenSHMEM provide one-sided communication primitives (e.g., put and get); Charm++ provides an asynchronous message passing mechanism; and Legion encapsulates all necessary data transfers within its runtime system and performs them transparently from the application.

The similar code length of the MPI_1S, MPI_2S and OSHM implementations is not surprising considering that these codes variants use similar memory and synchronization primitives, the same execution model, and offer only basic GPU support (i.e., they require explicit invocation of CUDA library calls for memory allocation and data transfer, kernel execution, and work-to-device mapping). MPI_1S saves a few LOC over MPI_2S because on most applications the use of one-sided communication allows removing some if-then-else blocks from the code. Since MPI requires the use of "windows" to implement one-sided communication, OSHM code is generally more compact than the MPI_1S counterpart. The only exception is the HIMENO benchmark. This application uses 3-D (3dimenion) data, and MPI offers primitives to handle 3-D data efficiently, while OSHM requires the programmer to manage these data structures explicitly.

Charm++'s memory and synchronization models contribute to its low LOC count. In Charm++, data distribution is performed using Charm++ high-level message passing primitives. However, the 
internals of the message passing mechanism are handled within the runtime and abstracted from the programmer. Similarly, data dependences and synchronizations are defined using simple primitives (e.g., when and atomic), but are otherwise completely handled by Charm++ runtime based on its internal dependences graphs. This allows compact code.

Legion's memory and synchronization model are responsible for its code length, the worst among the considered code variants. Recall that Legion requires the programmer to split the data space into logical regions, and associate to each of them an index and field space (encapsulating data structures definition and variables declaration), access privileges and coherence constraints. While this abstraction allows Legion to fully delegate data distribution and the required synchronization to its runtime, thus enabling data-aware scheduling and implicit data transfers and synchronizations, the definition of logical regions adds complexity to the code and contributes to its high LOC count.

Execution model - We classify existing parallel programming frameworks into two categories based on their PE execution model: Cooperative-Process (CP) and Task-Based (TB) frameworks. In the $\mathrm{CP}$ model, a computation is divided into parallel processes that execute for the entire lifetime of the application. The MPI-1 standard, OpenSHMEM and Legion implement the CP model. In the TB model, PEs do not necessarily remain active for the entire application lifetime, they can be spawned dynamically, and often execute small portions of code. MPI-2 and Charm++ implement the TB model.

The load balancing support provided by the programming framework affects code length and code complexity. Charm ++ and Legion provide automatic load balancing mechanisms within their runtime. MPI-1 and OpenSHMEM don't provide a flexible load balancing mechanism, but require programmers' to restructure their code so as to implement dynamic work partitioning and distribution. Since we aim to compare the programming frameworks without heavily restructuring the core of the computation, we did not encapsulate load balancing in the MPI-1 and OpenSHMEM code variants. MPI-2, on the other hand, provides dynamic process creation as a means to easily encapsulate load balancing in the application. Therefore, we have included load balancing through dynamic process creation in the MPI_DYN_1S and MPI_DYN_2S code variants.

In Charm++, load balancing (via dynamic task migration) can be activated with minimal code modifications. Task migration is performed based on profiling information collected at runtime and needs to be enabled at initialization. In addition, Charm++ provides primitives to interrupt chares, migrate them and resume their execution on the new site (namely, pauseForLB, AtSync, ResumeFromSync primitives). The few LOC added by the CHARM_LB code variant to the CHARM_NLB one contains the invocation of these task migration runtime primitives.

In the case of Legion, load balancing is active by default, and does not require any code modification to be enabled.

Since MPI requires the explicit integration of load balancing in the application, the LOC count of the MPI_DYN_2S and MPI_DYN_1S code variants is significantly higher than that of the basic MPI versions. The core idea of the MPI_DYN code variants is to balance the load by letting faster compute resources spawn new PEs dynamically to handle more work. To this end, these code variants include three kinds of PEs: master-PE, slave-PEs and workPEs. The master-PE is the first PE created (i.e., root PE). Each node
Table 2 Hardware Setup

\begin{tabular}{|c|c|l|}
\hline $\begin{array}{c}\text { Homog. } \\
\text { cluster }\end{array}$ & $\begin{array}{c}\mathbf{1 0} \\
\times\end{array}$ & CPU: 16-core AMD Opteron, 32 GB memory \\
GPU: 1 Geforce GTX 480 (Fermi), 480-cores, 1.5 GB memory \\
\hline
\end{tabular}

in the cluster has a slave-PE, whose goal is to monitor the resource utilization of the node and trigger load balancing actions. In particular, when the slave-PE detects node underutilization, it requests work to the master-PE, spawns a new work-PE, and assigns the work to it. At the end of the computation, the slave-PE reports work completion to the master-PE. The additional lines of code implement this interaction between the different PEs. The core computation of the application (which is common to the basic MPI code variants) is implemented within the work-PE code.

GPU Support - Since MPI requires programmers to specify all required GPU memory allocations, data transfers, kernel functions, and task-to-device mapping operations, it leads to the highest number of LOC devoted to GPU support. On the other hand, the GPU abstraction provided by Legion allows it to minimize the number of GPU-related LOC. OpenSHMEM and Charm++, which provide some basic GPU abstraction through wrapper functions, fall in the middle between MPI and Legion.

\section{Experimental Evaluation}

\subsection{Experiment setup}

Hardware and Software Setup - We run our experiments on a 10node homogeneous cluster equipped with NVidia GPUs. The clusters are interconnected through a 10GB Ethernet network. Table 2 shows the hardware configuration of the compute nodes. Red Hat Enterprise Linux Workstation 7.3, OpenMPI 2.0.1, CUDA 8.0, OpenSHMEM 1.2, Charm-6.7.0 and the latest Legion version are installed on every node.

Experiments - We performed two sets of experiments on the homogeneous cluster (horizontal and vertical scalability). We show the results reported on the MPI_2S, MPI_DYN_2S, OSHM, CHARM_NLB, CHARM_LB and LEGION code variants. Since the communication model used by MPI does not significantly affect performance, we omit showing MPI_1S and MPI_DYN_1S results.

Benchmark Applications - We selected five open-source applications with different arithmetic intensities, communication and synchronization patterns. Two of them (HIMENO and DMM) are memory-bounded, while three of them (NBODY, NW and CCT) are compute-bounded. All applications except CCT have regular synchronization patterns.

N-body simulation (NBODY) - NBODY simulates a dynamical system of particles, grouped into "bodies". The computation is performed iteratively. The computation is parallelized by partitioning the particles within a body across PEs, and it requires barrier synchronizations after the workload distribution phase and the end of iterations. We run 10 iterations on $30 \mathrm{k}-240 \mathrm{k}$ bodies and 20 iterations on $1200 \mathrm{k}$ bodies in our vertical and horizontal scalability experiments, respectively.

Dense Matrix Multiplication (DMM) - DMM multiplies two square matrices, with each PE handling one portion of the result matrix. DMM requires two synchronizations: one after the workload distribution phase and the other for gathering the final results. We used square matrices with dimension $600-4800$ and $8 \mathrm{k}$ in our vertical and horizontal scalability experiments, respectively.

Needleman-Wunsch (NW) [12] - NW is a dynamic programming algorithm widely used in bioinformatics for 
comparing biological sequences. The computation is parallelized by breaking genes into segments and distributing pairs of segments to PEs. In addition, workload splitting and gathering require two barriers. We used 80-1600 and 5000 pairs of genes of length 1000 in our vertical and horizontal scalability experiments, respectively.

Himeno (HIMENO) - HIMENO is a computational kernel that performs stencil computations on a 3-D grid of pressure values and is found in the simulation of incompressible fluids. HIMENO requires two synchronizations for data distribution and gathering, and one for data exchange. We used 5 datasets: xs (32x32x64 grids), $\mathrm{s}$ (64x64x128 grids), m (128x128x256 grids), 1 (128x128x256 grids) and $\mathrm{xl}(256 \times 256 \times 512$ grids): the first four in our vertical scalability experiment, and the last one in our horizontal scalability one.

Circuit Simulator (CCT) - CCT is a graph application that simulates the update of currents, charges and voltages in an electrical circuit. Edges within the graph represent wires. CCT performs three parallelizable tasks: current calculation, charge distribution and voltage update. Charge distribution requires data communication and atomic operations for shared wires. In all experiments, we run 10 iterations of CCT on circuit pieces consisting of 100 nodes and 150 wires, and we use 50-1600 and 2000 such circuit pieces in our vertical and horizontal experiments, respectively.

\subsection{Vertical Scalability Analysis}

We run the vertical scalability experiments on a single node of the homogenous cluster (see Table 2), progressively increasing the dataset size as indicated in Section 4.1. Figure 1 shows the results of these experiments. As could be expected, the execution time increases in all cases as the dataset size increases.

We make the following observations. First, on DMM, HIMENO and NW, CHARM_NLB performs significantly worse than OpenSHMEM and MPI. This is due to its runtime system's overhead. Recall that, in order to improve programmer productivity and application performance, Charm++'s runtime system maintains an internal SDAG [13] data structure and uses it to handle data transfers and synchronizations. This runtime overhead is paid off in case of applications with numerous synchronizations or complex synchronization patterns, such as NBODY and CCT. However, it affects negatively the performance of applications with infrequent synchronizations or simple synchronization patterns, such as DMM, HIMENO and NW. Second, Legion exhibits the worst performance and scaling behavior across all datasets and applications. This is because, to completely hide data transfers and synchronizations from the programmer, Legion has an even higher runtime overhead than Charm++. Third, Legion fails to run on larger datasets (e.g., $\mathrm{DMM} / 4800 \mathrm{x} 4800, \mathrm{HIMENO} / \mathrm{m}$ and larger, $\mathrm{CCT} />200$ circuit pieces). This is because Legion's runtime system duplicates data belonging to logical regions that are shared across PEs, leading to higher memory requirements than the other programming frameworks. On large datasets, Legion's memory footprint exceeds the memory capacity of the compute node.

\subsection{Horizontal Scalability Analysis}

We run our horizontal scalability experiments on the 10-node homogeneous cluster of Table 2. Unfortunately, we have no administrative privileges on this cluster, and its resource manager's configuration conflicts with the inter-node communication settings required by Legion, preventing us from performing multi-node experiments on this framework. Therefore, we limit our horizontal scalability discussion to MPI, OpenSHMEM and Charm++. For this set of experiments, we fix the dataset size (see Section 4.1) and progressively increase the number of compute nodes used from 2 to 10. For each node configuration, we vary the number of processes from 20 to 64 . Figure 2 reports the horizontal scalability results.

We make the following observations. First, performance improves in all cases almost linearly as we increase the number of compute nodes. Hence, all frameworks show good horizontal scalability on small clusters.

Second, in this set of experiments Charm++ performs similarly to MPI and OpenSHMEM. On HIMENO and CCT the performance of the three frameworks are almost identical. On DMM and NBODY, although there is some variation, for some processes settings Charm++ performs similarly (or better) than MPI. The only application for which Charm++ shows worse vertical scalability is NW, which is compute bound and so does not benefit from runtime data transfers optimizations. The better behavior of Charm++ as compared to the single-node vertical scalability experiments is due to the larger dataset used here and the distributed environment. The increased amount of work amortizes Charm++ runtime overhead. In addition, runtime optimizations (such as the ones performed by Charm++) are more effective in distributed environments, where synchronizations and data transfers are more costly.

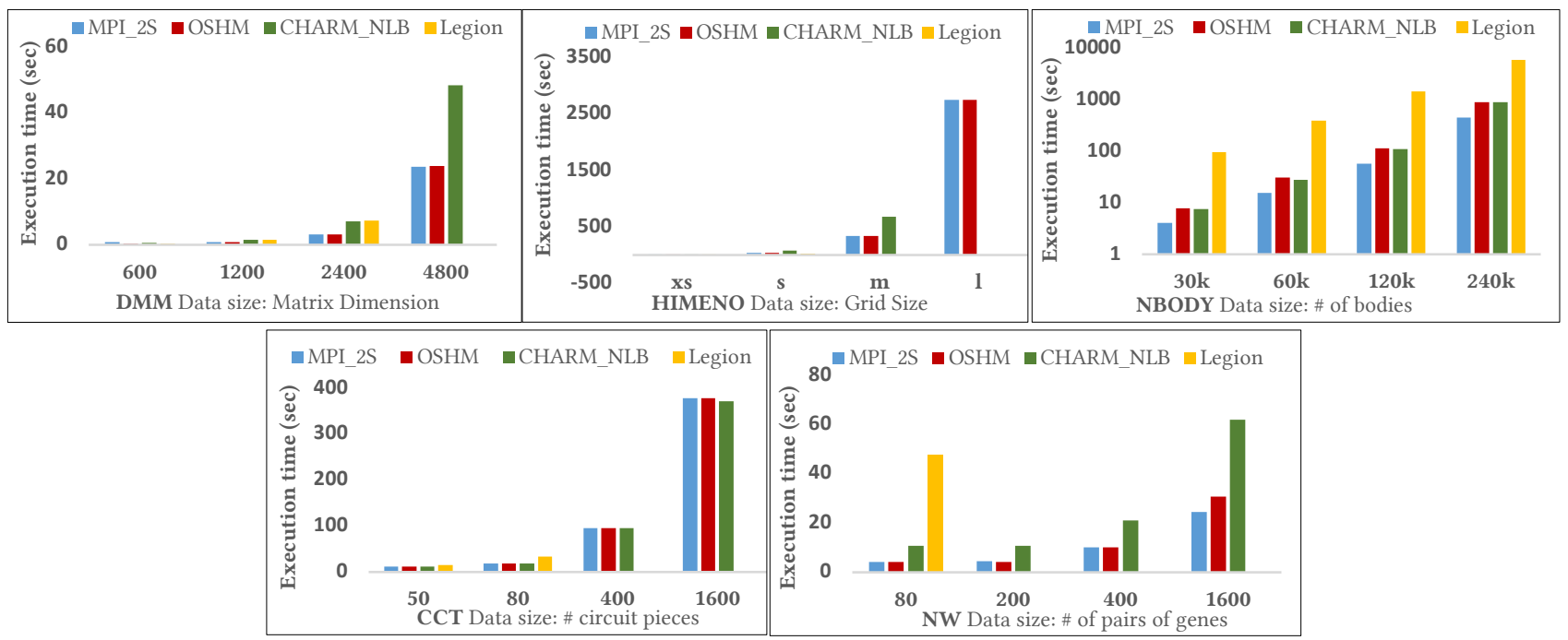

Figure 1 Vertical Scalability Results (note: NBODY results are in logarithmic scale) 
Third, the effect of increasing the number of processes on a given cluster setting is not consistent across applications and implementations. HIMENO and CCT are not significantly affected by the increase in the number of processes. For the memoryintensive DMM, increasing the number of processes can help hiding memory latencies. For compute-intensive applications (like NBODY, NW and CCT) few processes are enough to guarantee good performance, and increasing the number of processes can cause more synchronization and process management overhead.

Finally, we were unable to run 2-node experiments on NW. This is because NW's memory footprint exceeds the memory capacity of two nodes.

\section{Related Work}

Previous works have performed programmability and performance comparisons between various parallel programming frameworks. Lin et al. [14] have compared shared memory and non-shared memory-based programming models on shared memory machines from a programmability standpoint. Podobas et al. [15] have evaluated task-based parallel programming models for shared memory systems, including Cilk++ [16], OpenMP and Wool [17]. Both works are limited to a single machine instead of a cluster. Daga et al. [18] have compared three parallel programming models for heterogeneous systems, namely: OpenCL [19], C++ AMP [20] and OpenACC [21], and they have evaluated them on discrete and integrated GPUs using a diverse set of applications. Their focus is on one- or two-node GPU systems, while our focus is on the use of established and emerging programming frameworks for small-, medium- and large-scale systems that include GPUs.

Previous efforts have led to the design of more programming frameworks for systems that comprise hardware accelerators. Augonnect et al. [8] have designed StarPU, a computing platform for heterogeneous systems that provide a runtime system with a unified high-level interface for different accelerators (e.g., GPUs, Cell/BE SPUs). StarPU follows a task-based execution model and offers common scheduling and load balancing policies. Planas et al. [9] have developed StarSs, another task-based programming model that uses pragma annotations. Their goal is to introduce automatic tasklevel parallelism on different accelerator architectures with parallel task extraction and scheduling, data dependences detection and hierarchical task-level support. Extending our analysis to these frameworks can be subject of future work.

\section{Conclusion}

In this work, we have characterized the memory model, the synchronization model, the execution model and the GPU support offered by MPI, OpenSHMEM, Charm++ and Legion and we have studied how these features affect programmability and performance of applications in homogeneous cluster setting. From the programmability standpoint, MPI-2 and OpenSHMEM are similar in that they support one-sided communication and provide explicit memory management and synchronization primitives. In addition, MPI provides dynamic process creation, which facilitate encapsulating load balancing within the application. Charm++ provides an object-oriented programming model with high-level memory management and synchronization primitives, and delegates to the runtime data dependences handling and the use of low-level memory transfer and synchronization mechanisms. Finally, Legion provides a data-centric programming model, requires the programmer to partition the data into logical regions with associated access privileges and coherence constraints, and abstracts from the programmer any data transfers and synchronization required to implement those constraints by delegating them to the runtime, which performs data-aware scheduling.

Delegating memory management, synchronization handling and load balancing to the runtime system hurts Charm++ and Legion performance and scalability. By leaving more control to the programmer, MPI and OpenSHMEM allow low-overhead runtime libraries that lead to good and more easily tunable performance. On a single-node system, Charm++ runtime overhead affects performance, but does so less dramatically than Legion's runtime overhead. Charm's overhead is amortized or hidden on multi-node environments and on large datasets. MPI dynamic process creation allows easily integrating load balancing mechanisms in the applications, but these mechanisms are less effective on code with complex synchronization patterns.

\section{Acknowledgments}

This work has been supported by National Science Foundation award CCF-1728850.
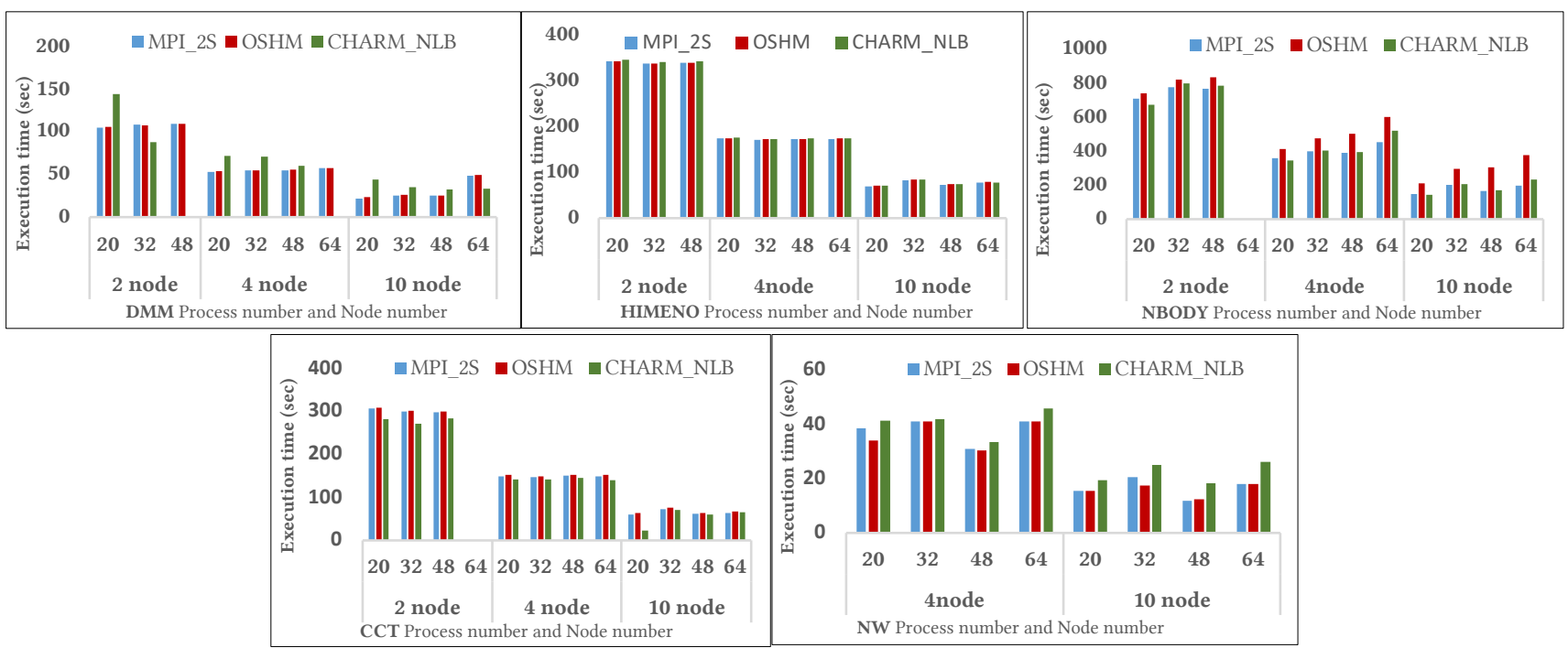

Figure 2 Horizontal Scalability Results 


\section{References}

[1] E. Gabriel, G. E. Fagg, G. Bosilca, T. Angskun, J. J. Dongarra, S. J. M., et al., "Open MPI: Goals, Concept, and Design of a Next Generation MPI Implementation," presented at the Proceedings, 11th European PVM/MPI Users' Group Meeting, 2004.

[2] T. Curtis, S. Pophale, B. Chapman, S. Poole, J. Kuehn, O. Hernandez, et al. (2014) OpenSHMEM Specification 1.1 Final. Available: http://www.openshmem.org/

[3] Unified Parallel C. Available: https://upc-lang.org/

[4] The Chapel Parallel Programming Language. Available: http://chapel.cray.com/

[5] X10: Performance and Productivity at Scale. Available: http://x10-lang.org/

[6] L. V. Kale and S. Krishnan, "CHARM++: a portable concurrent object oriented system based on C++," presented at the ACM Sigplan Notices, 1993.

[7] M. Bauer, S. Treichler, E. Slaughter, and A. Aiken, "Legion: expressing locality and independence with logical regions," presented at the Proceedings of the International Conference on High Performance Computing, Networking, Storage and Analysis, Salt Lake City, Utah, 2012.

[8] C. Augonnet, S. Thibault, R. Namyst, and P. A. Wacrenier, "StarPU: a unified platform for task scheduling on heterogeneous multicore architectures," Concurrency and Computation: Practice and Experience, vol. 23, pp. 187-198, 2011.

[9] J. Planas, R. M. Badia, E. Ayguadé, and J. Labarta, "Hierarchical task-based programming with StarSs," The International fournal of High Performance Computing Applications, vol. 23, pp. 284-299, 2009.

[10] B. Acun, A. Gupta, N. Jain, A. Langer, H. Menon, E. Mikida, et al., "Paralle programming with migratable objects: charm++ in practice," presented at the Proceedings of the International Conference for High Performance Computing, Networking, Storage and Analysis, New Orleans, Louisana, 2014.

[11] M. T. Nelson, W. Humphrey, A. Gursoy, A. Dalke, L. V. Kalé, R. D. Skeel, et al., "NAMD: a parallel, object-oriented molecular dynamics program," The International Gournal of Supercomputer Applications and High Performance Computing, vol. 10, pp. 251-268, 1996.

[12] D. Li, K. Sajjapongse, H. Truong, G. Conant, and M. Becchi, "A Distributed CPUGPU Framework for Pairwise Alignments on Large-Scale Sequence Datasets," presented at the Proceedings of the 24th IEEE International Conference on Application-specific Systems, Architectures and Processors (ASAP), Ashburn, VA, 2013.

[13] L. V. Kale and K. S., "Charm++: Parallel Programming with Message-Driven Objects," presented at the Parallel Programming using C++, 1996.

[14] C. Lin and L. Snyder, "A comparison of programming models for shared memory multiprocessors," presented at the ICPP (2), 1990.

[15] A. Podobas, M. Brorsson, and K.-F. Faxén, "A comparison of some recent task-based parallel programming models," presented at the 3rd Workshop on Programmability Issues for Multi-Core Computers, 2010.

[16] H. Vandierendonck, "The Cilk and Cilk++ Programming Languages," in Multicore Computing: Algorithms, Architectures, and Applications, ed, 2013, p. 91.

[17] K.-F. Faxén. (2015). Wool user's guide. Available: https://www.sics.se/ kff/wool/users-guide.pdf

[18] M. Daga, Z. S. Tschirhart, and C. Freitag, "Exploring Parallel Programming Models for Heterogeneous Computing Systems," presented at the Workload Characterization (IISWC), 2015 IEEE International Symposium on, 2015.

[19] J. E. Stone, D. Gohara, and G. Shi, "OpenCL: A parallel programming standard for heterogeneous computing systems," presented at the Computing in science \& engineering, 2010

[20] T. M. Evans and K. T. Clarno, "C++ Coding Standards for the AMP Project," in ORNL-TM/2009/240, Rev. 0, Oak Ridge National, Laboratory, Oak Ridge, Tenn, ed, 2009.

[21] S. Wienke, P. Springer, C. Terboven, and D. an Mey, "OpenACC-first experiences with real-world applications," presented at the Euro-Par 2012 Parallel Processing, 2012 . 